\title{
Edge Theoretic Extended Contractions and Their Applications
}

\author{
R. Rajagopalan $\mathbb{D}^{1},{ }^{1}$ Ekta Tamrakar, ${ }^{2}$ Fahad. S. Alshammari $\mathbb{D},{ }^{1}$ H. K. Pathak, ${ }^{2}$ \\ and Reny George $\mathbb{i}^{1,3}$ \\ ${ }^{1}$ Department of Mathematics, College of Science and Humanities at Al-Kharj, Prince Sattam Bin Abdulaziz University, \\ Al-Kharj 11942, Saudi Arabia \\ ${ }^{2}$ School of Studies in Mathematics, Pt. Ravishankar Shukla University, Raipur 492010, India \\ ${ }^{3}$ PG Department of Mathematics and Computer Science, St. Thomas College, Bhilai, Chhattisgarh State, India
}

Correspondence should be addressed to R. Rajagopalan; r.gopalan@psau.edu.sa and Reny George; renygeorge02@yahoo.com

Received 13 September 2021; Revised 15 October 2021; Accepted 18 October 2021; Published 9 November 2021

Academic Editor: Huseyin Isik

Copyright ( 2021 R. Rajagopalan et al. This is an open access article distributed under the Creative Commons Attribution License, which permits unrestricted use, distribution, and reproduction in any medium, provided the original work is properly cited.

Edge theoretic extended contractions are introduced and coincidence point theorems and common fixed-point theorems are proved for such contraction mappings in a metric space endowed with a graph. As further applications, we have proved the existence of a solution of a nonlinear integral equation of Volterra type and given a suitable example in support of our result.

\section{Introduction and Preliminaries}

The celebrated Banach contraction principle is a motivation for many fixed-point theorems. It guarantees the existence and uniqueness of solution of various equations arising in mathematics. The initial generalizations of Banach's result came up in the form of Kannan's contraction, Chatterjea's contraction, Reich's contraction, Ciric's contraction, HardyRoger's contraction, and Ciric's quasicontraction. Among these, Ciric's quasicontraction is the most general form in the sense that any mapping which does not satisfy Ciric's quasicontraction does not satisfy any of the previously mentioned contractions. Further, these results have been widely investigated and many interesting applications have been found by many authors (see [1-7]). F-contraction and fixed-point theorem for $F$-contraction mappings were introduced by Wardowski [8] as a generalisation of the Banach contraction principle.

Definition 1 (see [8]). Consider the collection of functions $F:(0, \infty) \longrightarrow \mathbb{R}$ satisfying the following:

$\left(F_{1}\right) F$ is strictly increasing

$\left(F_{2}\right)$ If $\left\{\alpha_{n}\right\} \subset(0, \infty)$ is a sequence, then $\lim _{n \longrightarrow \infty} \alpha_{n}=0$ iff $\lim _{n \rightarrow \infty} F\left(\alpha_{n}\right)=-\infty$

$\left(F_{2}\right)$ There exists $k \in(0,1)$ such that $\lim _{\gamma \longrightarrow 0^{+}} \gamma^{k} F(\gamma)=0$
An operator $T: X^{i}, d_{i} \longrightarrow X^{i}$ is an $\mathscr{F}$-contraction if we can find $\tau>0$ such that

$\forall x^{i}, y^{i} \in X^{i}, d_{i}\left(T x^{i}, T y^{i}\right)>0 \Longrightarrow \tau+F\left(d_{i}\left(T x^{i}, T y^{i}\right)\right) \leq F\left(d_{i}\left(x^{i}, y^{i}\right)\right)$.

Later, the concept of $F$-weak contraction and ordered $F$ -contractions was introduced by Wardowski and Van Dung [9] and Durmaz et al. [10], respectively. In 2016, Sawangsup et al. [11] extended the $F$-contraction using a relation theoretic approach which was later generalised by Imdad et al. [12] and Alfaqih et al. [13]. Espinola and Kirk [14] introduced graph theory in fixed-point theory, and Jachymski [15] continued this idea by using different views thereby introducing the $G$-contraction and proved fixed-point theorem for a $G$-contraction mapping. These ideas were further extended and generalised by [16-24].

It is interesting to note that all these contraction conditions ensure the existence of a unique fixed point or common fixed point of the mappings under consideration. However, it is observed that a mapping which possesses nonunique fixed points does not satisfy the above contractions, for if $x^{i}$ and $y^{i}$ are any two fixed points of a self-map $T^{i}$ of a metric space $\left(X^{i}, d^{i}\right)$, then 


$$
\begin{aligned}
& d^{i}\left(T^{i} x^{i}, T^{i} y^{i}\right)=d^{i}\left(x^{i}, y^{i}\right) \\
& =\max \left\{d^{i}\left(x^{i}, y^{i}\right), d^{i}\left(x^{i}, T^{i} x^{i}\right), d^{i}\left(y^{i}, T^{i} y^{i}\right), \frac{d^{i}\left(x^{i}, T^{i} y^{i}\right)+d^{i}\left(y^{i}, T^{i} x^{i}\right)}{2}\right\}, \\
& d^{i}\left(T^{i} x^{i}, T^{i} y^{i}\right)=d^{i}\left(x^{i}, y^{i}\right) \\
& \quad=\max \left\{d^{i}\left(x^{i}, y^{i}\right), d^{i}\left(x^{i}, T^{i} x^{i}\right), d^{i}\left(y^{i}, T^{i} y^{i}\right), d^{i}\left(x^{i}, T^{i} y^{i}\right), d^{i}\left(y^{i}, T^{i} x^{i}\right)\right\},
\end{aligned}
$$

and thus, we see that $T^{i}$ does not satisfy Ciric's quasicontraction, Wardowski's F-contraction, and Wardowski and Van Dung's $F$-weak contraction. Thus, these contraction conditions cannot be used to prove the existence of nonunique fixed points of a function defined in a metric space. On the other hand, many equations obtained by modeling various problems of engineering and science need not necessarily have a unique solution. Thus, it becomes meaningful to obtain extended forms of above contractions which will ensure the existence of nonunique fixed points of self-maps defined in a metric space.

Motivated by this fact, in this paper, we have introduced extended $\mathscr{J} \mathscr{W}$-contraction (Jungck-Wardowski contraction), extended $\mathscr{C} \mathscr{W}$-contraction (Ciric-Wardowski contraction), and extended $\mathscr{C} \mathscr{W} Q$-contraction (Ciric-Wardowski quasicontraction) and established fixed-point theorems which will ensure the existence of nonunique fixed points of a self-map and coincidence points of a pair of self-maps, respectively, in a metric space endowed with a graph. As an application of our result, we have also proven the existence of solution of a nonlinear integral equation of Volterra type.

Throughout this paper, we consider the metric space $\left(X^{j}, d_{j}\right)$ to be endowed with the graph $G=(V(G), E(G))$, $V(G)=X^{j}$, and $\Delta \subseteq E(G) ; \Delta=\left\{\left(x^{j}, x^{j}\right): x^{j} \in X^{j}\right\}$.

Definition 2 (see [15]). A sequence $\left\{x_{n}^{j}\right\} \subseteq X^{j}$ is edgepreserving if $\left(x_{n}^{j}, x^{j}{ }_{n+1}\right) \in E(G)$ for all $n \in \mathbb{N}_{0}$.

Definition 3. Let $g: X^{j} \longrightarrow X^{j}$. A sequence $\left\{x_{n}^{j}\right\} \subseteq X^{j}$ is $g$ -edge-preserving if $\left(g x_{n}^{j}, g x^{j}{ }_{n+1}\right) \in E(G)$ for all $n \in \mathbb{N}_{0}$.

Definition 4. $T: X^{j} \longrightarrow X^{j}$ is edge-preserving if $\left(x^{j}, y^{j}\right) \in E$ $(G)$ implies $\left(T x^{j}, T y^{j}\right) \in E(G)$.

Definition 5. T, $g: X^{j} \longrightarrow X^{j}$ is $g$-edge-preserving if for all $x^{j}, y^{j} \in X,\left(g x^{j}, g y^{j}\right) \in E(G)$ implies $\left(T x^{j}, T y^{j}\right) \in E(G)$.

Definition 6 (see [15]). $\left(X^{j}, d_{j}\right)$ is edge-complete if every edge-preserving Cauchy sequence in $X^{j}$ converges to some point in $X^{j}$.

Definition 7 (see [15]). $T: X^{j} \longrightarrow X^{j}$ is edge-continuous at $x^{j}$ if $\left\{x_{n}^{j}\right\} \longrightarrow x^{j}$ implies $\left\{T x^{j}{ }_{n}\right\} \longrightarrow T x^{j}$ for any edgepreserving sequence $\left\{x_{n}^{j}\right\} \subseteq X^{j}$. If $T$ is edge-continuous at all $x^{j} \in X^{j}$, then $T$ is an edge-continuous mapping.

Definition 8. Let $T, g: X^{j} \longrightarrow X^{j}$ and $x^{j} \in X^{j}$. We say that $T$ is $g$-edge continuous at $x^{j}$ if $\left\{g x^{j}{ }_{n}\right\} \longrightarrow g x^{j}$ implies $\left\{T x_{n}^{j}\right\}$ $\longrightarrow T x^{j}$ for any edge-preserving sequence $\left\{x_{n}^{j}\right\} \subseteq X^{j}$. If $T$ is $g$-edge continuous at all $x^{j} \in X^{j}$, then $T$ is an $g$-edge continuous mapping.

Definition 9. $(T, g)$ is edge-compatible if and only if for any sequence $T$ and $g$ edge-preserving sequence $\left\{x_{n}^{j}\right\} \subseteq$ $X, \lim _{n \longrightarrow \infty} g x_{n}^{j}=\lim _{n \longrightarrow \infty} T x_{n}^{j}=x \in X^{j}$ implies $\lim _{n \longrightarrow \infty}$ $d_{j}\left(g T x_{n}^{j}, T g x_{n}^{j}\right)=0$.

We will use the following lemmas taken from $[25,26]$ :

Lemma 10. (see [25]). Let $M$ be a nonempty set and $g: M$ $\longrightarrow M$. Then, there exists a subset $S \subseteq M$ such that $g(s)=g$ $(M)$ and $g: S \longrightarrow S$ is one-one.

Lemma 11 (see [26]). Let $\left\{x_{n}^{j}\right\}$ be a sequence in metric space $\left(X^{j}, d_{j}\right)$ such that $\lim _{n \longrightarrow+\infty} d_{j}\left(x_{n}^{j}, x_{n+1}^{j}\right)=0$. If $\left\{x_{n}^{j}\right\}$ is not Cauchy in $\left(X^{j}, d_{j}\right)$, then there exist $\xi>0$ and sequences $\left\{n_{k}\right\}$ and $\left\{p_{k}\right\}$ in $\mathbb{N}$ such that $n_{k}>p_{k}>k$, and the sequences

$$
\begin{gathered}
\left\{d_{j}\left(x_{n_{k}}^{j}, x_{p_{k}}^{j}\right)\right\},\left\{d_{j}\left(x_{n_{k}+1}^{j}, x_{p_{k}}^{j}\right)\right\},\left\{d_{j}\left(x_{n_{k}}^{j}, x_{p_{k}-1}^{j}\right)\right\}, \\
\left\{d_{j}\left(x_{n_{k}+1}^{j}, x_{p_{k}-1}^{j}\right)\right\},\left\{d_{j}\left(x_{n_{k}+1}^{j}, x_{p_{k}+1}^{j}\right)\right\},
\end{gathered}
$$

tend to be $\xi^{+}$, as $k \longrightarrow+\infty$.

\section{Edge Theoretic Extended Contractions}

Let $\mathbb{F}$ be the collection of all nondecreasing continuous functions $\mathscr{F}:(0, \infty) \longrightarrow \mathbb{R}$.

Example 1. Some examples of function belonging to the class $\mathbb{F}$ are

$$
\begin{aligned}
& \mathscr{F}(y)=y^{2}, \\
& \mathscr{F}(y)=\ln y, \\
& \mathscr{F}(y)=y-\frac{1}{y}, \\
& \mathscr{F}(y)=\ln \left(\frac{y}{3}+\sin y\right) .
\end{aligned}
$$

Let $A \subset[0, \infty)$ and $\Xi$ be the collection of all continuous functions $\xi: A \times A \longrightarrow[0, \infty)$ satisfying the following:

(i) $\alpha=0$ or $\beta=0$ implies $\xi(\alpha, \beta)=0$

(ii) $\alpha>0$ and $\beta>0$ implies $\xi(\alpha, \beta)>0$

$$
\sup _{\alpha, \beta \in A} \xi(\alpha, \beta)=\zeta>0
$$

Some examples of function $\xi$ are as follows:

Example 2. 
(i) $\xi(\alpha, \beta)=k \cdot \alpha \beta$, for some $k>0$

(ii) $\xi(\alpha, \beta)=\min \{\alpha, \beta\}$

(iii) $\xi(\alpha, \beta)=\alpha /(1+\ln \beta)$

(iv) $\xi(\alpha, \beta)=(\alpha+\beta) /(1+\ln (\alpha \beta))$

(v) $\xi(\alpha, \beta)=\alpha \beta(\alpha+\beta)$

(vi) $\xi(\alpha, \beta)=\alpha \beta /(1+\alpha \beta)$

(vii) $\xi(\alpha, \beta)=\ln (1+K \cdot \min \{\alpha, \beta\}$

Let $\Theta$ be the family of all functions $\theta:[0, \infty) \longrightarrow R$ which satisfy the following conditions:

$\left(\theta_{1}\right) \theta$ is strictly increasing

$\left(\theta_{2}\right) \theta(t)=0$ iff $t=0$

$\left(\theta_{3}\right) \sup _{t>0} \theta(t)=\lambda$ for some $\lambda>0$

Example 3. Some examples of elements of $\Theta$ are

$$
\begin{aligned}
& \theta(t)=\frac{t}{1+t}, \\
& \theta(t)=\ln \left(1+\frac{t}{1+t}\right), \\
& \theta(t)=\frac{t}{1+\ln (1+t)} .
\end{aligned}
$$

where

Definition 12. A pair of mappings $T, g: X^{j} \longrightarrow X^{j}$ is an $\xi$ -extended $\mathscr{J} \mathscr{W}$-contraction pair if we can find $\tau>0, F \in \mathscr{F}$, $\xi \in \Xi$, and $L \geq 0$ such that for all $x^{j}, y^{j} \in X^{j}$,

$$
\begin{aligned}
d_{j}\left(T x^{j}, T y^{j}\right) & >0 \Longrightarrow \tau+F\left(d_{j}\left(T x^{j}, T y^{j}\right)\right) \\
& \leq \mathscr{F}\left(d_{j}\left(g x^{j}, g y^{j}\right)\right)+L \xi\left(d_{j}\left(g y^{j}, T x^{j}\right), d_{j}\left(g x^{j}, T y^{j}\right)\right),
\end{aligned}
$$

Definition 13. A pair of mappings $T, g: X^{j} \longrightarrow X^{j}$ is an $\xi$ -extended $\mathscr{C} \mathscr{W}$-contraction pair if we can find $\tau>0, F \in \mathscr{F}$, $\xi \in \Xi$, and $L \geq 0$ such that for all $x^{j}, y^{j} \in X^{j}$,

$$
\begin{aligned}
d_{j}\left(T x^{j}, T y^{j}\right) & >0 \Longrightarrow \tau+F\left(d_{j}\left(T x^{j}, T y^{j}\right)\right) \\
& \leq \mathscr{F}\left(M^{j}\left(x^{j}, y^{j}\right)\right)+L \xi\left(d_{j}\left(g y^{j}, T x^{j}\right), d_{j}\left(g x^{j}, T y^{j}\right)\right)
\end{aligned}
$$

$$
M^{j}\left(x^{j}, y^{j}\right)=\max \left\{d_{j}\left(g x^{j}, g y^{j}\right), d_{j}\left(g x^{j}, T x^{j}\right), d_{j}\left(g y^{j}, T y^{j}\right), \frac{d_{j}\left(g x^{j}, T y^{j}\right)+d_{j}\left(g y^{j}, T x^{j}\right)}{2}\right\} .
$$

Definition 14. A pair of mappings $T, g: X^{j} \longrightarrow X^{j}$ is an $\xi$ -extended $\mathscr{C} \mathscr{W} Q$-contraction pair provided that there is a $\tau>0, F \in \mathscr{F}, \xi \in \Xi$, and $L \geq 0$ such that for all $x^{j}, y^{j} \in X^{j}$,

$$
\begin{aligned}
d_{j}\left(T x^{j}, T y^{j}\right) & >0 \Longrightarrow \tau+F\left(d_{j}\left(T x^{j}, T y^{j}\right)\right) \\
& \leq \mathscr{F}\left(M^{j^{*}}\left(x^{j}, y^{j}\right)\right)+L \xi\left(d_{j}\left(g y^{j}, T x^{j}\right), d_{j}\left(g x^{j}, T y^{j}\right)\right),
\end{aligned}
$$

where

$$
\begin{aligned}
M^{j^{*}}\left(x^{j}, y^{j}\right)= & \max \left\{d_{j}\left(g x^{j}, g y^{j}\right), d_{j}\left(g x^{j}, T x^{j}\right), d_{j}\right. \\
& \left.\cdot\left(g y^{j}, T y^{j}\right), d_{j}\left(g x^{j}, T y^{j}\right), d_{j}\left(g y^{j}, T x^{j}\right)\right\} .
\end{aligned}
$$

Definition 15. In Definitions 12, 13, and 14, if conditions (7), (8), and (10) are satisfied only for all $x^{j}, y^{j} \in X^{j}$ with $\left(x^{j}, y^{j}\right)$ $\in E(G)$, then the pair $(T, g)$ is an $\xi$-extended $\mathscr{J} \mathscr{W}$-edge contraction, $\xi$-extended $\mathscr{C} \mathscr{W}$-edge contraction, and $\xi$ -extended $\mathscr{C} \mathscr{W} \mathscr{Q}$-edge contraction, respectively.
Definition 16. T, $g: X^{j} \longrightarrow X^{j}$ is a $\theta$-extended $\mathscr{J} \mathscr{W}$-edge contraction if we can find $\tau>0, F \in \mathscr{F}$, and $\theta \in \Theta$ such that for all $x^{j}, y^{j} \in X^{j}$ with $\left(g x^{j}, g y^{j}\right) \in E(G)$,

$$
\begin{aligned}
d_{j}\left(T x^{j}, T y^{j}\right) & >0 \Longrightarrow \tau+F\left(d_{j}\left(T x^{j}, T y^{j}\right)\right) \\
& \leq \mathscr{F}\left(d_{j}\left(g x^{j}, g y^{j}\right)\right)+L \theta\left(d_{j}\left(g y^{j}, T x^{j}\right)\right) .
\end{aligned}
$$

Definition 17. A pair of mappings $T, g: X^{j} \longrightarrow X^{j}$ is a $\theta$ -extended $\mathscr{C} \mathscr{W}$-edge contraction if we can find $\tau>0, F \in$ $\mathscr{F}$, and $\theta \in \Theta$ such that

$$
\begin{aligned}
d_{j}\left(T x^{j}, T y^{j}\right) & >0 \Longrightarrow \tau+F\left(d_{j}\left(T x^{j}, T y^{j}\right)\right) \\
& \leq \mathscr{F}\left(M^{j}\left(x^{j}, y^{j}\right)\right)+L \theta\left(d_{j}\left(g y^{j}, T x^{j}\right)\right),
\end{aligned}
$$

for all $x^{j}, y^{j} \in X^{j}$ with $\left(g x^{j}, g y^{j}\right) \in E(G)$ and $\left.M^{j}\left(x^{j}, y^{j}\right)\right)$, is as in (9).

If $g=I$ in the above definitions, then $T$ is an $\xi$-extended $F$-contraction mapping, $\xi$-extended $\mathscr{C} \mathscr{W}$-contraction mapping, $\theta$-extended $\mathscr{J} \mathscr{W}$-edge contraction mapping, and $\theta$ -extended $\mathscr{C} \mathscr{W}$-edge contraction mapping, respectively. 
Property $(*)$. The space $\left(X^{j}, d_{j}\right)$ is said to have property $(*)$ if for any edge-preserving sequence $\left\{x_{n}^{j}\right\} \in X$ such that $\left\{x_{n}^{j}\right\} \longrightarrow x$; there exists a subsequence $\left\{x_{n_{k}}^{j}\right\}$ of $\left\{x_{n}^{j}\right\}$ such that $\left.\left(x_{n_{k}}^{j}, x\right) \in E(G)\right|_{X}$ for all $k \in \mathbb{N}_{0}$

Example 4. Let $X=[0,1] \bigcup\{2\}, d_{j}\left(x^{i}, y^{j}\right)=\left|x^{i}-y^{j}\right|$, and $T$ $x^{i}=x^{i} / 8$ for all $x^{i} \in X$. Then, at $x^{i}=0$ and $y^{j}=2, T$ does not satisfy the conditions of Ciric's quasicontraction, Wardowski's $F$-contraction, and Wardowski and Van Dung's $F$ - weak contraction. However, $T$ is an $\xi$-extended $F$-contraction with $\tau=\ln (2)$, as shown below:

Let $F:(0, \infty) \longrightarrow \mathbb{R}$ be defined by

$$
F(t)=\ln (t)
$$

and $\xi(\alpha, \beta)=\ln (1+K \cdot \min \{\alpha, \beta\})$.

Case 1. $x^{i}, y^{j} \in[0,1]$. Clearly,

$$
\begin{aligned}
d_{j}\left(T x^{i}, T y^{j}\right) & =\frac{1}{8}\left|x^{i^{4}}-y^{j^{4}}\right| \leq \frac{1}{8}\left|x^{i}-y^{j} \| x^{i}+y^{j}\right|\left|x^{i^{2}}+y^{j^{2}}\right| \\
& \leq \frac{1}{4}\left|x^{i}-y^{j}\left\|x^{i}+y^{j}\right\|<\frac{1}{2}\right| x^{i}-y^{j} \mid \leq \frac{1}{2} d_{j}\left(x^{j}, y^{j}\right) .
\end{aligned}
$$

Then, we have $\ln \left(d_{j}\left(T x^{i}, T y^{j}\right)\right)<\ln \left(1 / 2 d_{j}\left(x^{j}, y^{j}\right)\right)$ or

$\ln 2+\ln \left(d_{j}\left(T x^{i}, T y^{j}\right)\right)<\ln \left(d_{j}\left(x^{j}, y^{j}\right)\right)+L \xi\left(d_{j}\left(g y^{j}, T x^{j}\right), d_{j}\left(g x^{j}, T y^{j}\right)\right)$.

Case 2. $x^{i} \in[0,1]$ and $y^{j}=2$. Note that in this case, $d_{j}\left(x^{j}\right.$, $\left.y^{j}\right) \geq 1$.

$$
\begin{aligned}
d_{j}\left(T x^{i}, T y^{j}\right) & =\left|\frac{x^{i^{4}}}{8}-2\right| \leq \frac{1}{2}+2 \min \left\{\left|x^{i}-2\right|,\left|2-\frac{x^{i^{4}}}{8}\right|\right\} \\
& \left.\Longrightarrow d_{j}\left(T x^{i}, T y^{j}\right)\right) \\
& \leq \frac{1}{2} d_{j}\left(x^{j}, y^{j}\right)\left(1+8 \min \left\{\left|x^{i}-2\right|,\left|2-\frac{x^{i^{4}}}{8}\right|\right\}\right) \\
& \Longrightarrow \ln \left(d_{j}\left(T x^{i}, T y^{j}\right)\right) \\
& \leq-\ln 2+\ln \left(d_{j}\left(x^{j}, y^{j}\right)\right)+\ln \left(1+8 \min \left\{\left|x^{i}-2\right|,\left|2-\frac{x^{i^{4}} \mid}{8}\right|\right\}\right) \\
& \Longrightarrow \ln 2+\ln \left(d_{j}\left(T x^{i}, T y^{j}\right)\right) \\
& \leq \ln \left(d_{j}\left(x^{j}, y^{j}\right)\right)+\ln \left(1+8 \min \left\{\left|x^{i}-2\right|,\left|2-\frac{x^{i^{4}}}{8}\right|\right\}\right) \\
& \Longrightarrow \ln 2+F\left(d_{j}\left(T x^{i}, T y^{j}\right)\right) \\
& \leq F\left(d_{j}\left(x^{j}, y^{j}\right)\right)+\xi\left(1+8 \min \left\{d_{j}\left(x^{j}, T y^{j}\right), d_{j}\left(y^{j}, T x^{j}\right)\right\}\right) .
\end{aligned}
$$

Example 5. Let $X^{j}=[0, \infty), d_{j}\left(x^{j}, y^{j}\right)=\left|x^{j}-y^{j}\right|, E(G)=\{(n$, $n),(n, n+1): n=0,1,2,3, \cdots\}$, and $T, g: X^{j} \longrightarrow X^{j}$ be given by

$$
T x^{j}=\left(\begin{array}{ll}
0, & \text { if } 0 \leq x^{j} \leq 1, \\
x^{j}-1, & \text { if } x^{j} \geq 1,
\end{array}\right.
$$

$g x^{j}=x^{j}+\left(n+1-x^{j}\right)\left(x^{j}-n\right), \quad$ whenever $n \leq x^{j} \leq n+1$.

Let $F:(0, \infty) \longrightarrow \mathbb{R}$ be defined by

$$
F(t)=t-\frac{1}{t}
$$

and $\theta \in \Theta$ be defined by $\theta(t)=t /(t+1)$. Then,

$$
\begin{aligned}
\tau+ & F\left(d_{j}(T(n), T(n+1)) \leq F\left(d_{j}(g(n), g(n+1))\right.\right. \\
& +L \theta\left(d_{j}(g(n+1), T(n))\right. \\
\Longrightarrow & \tau+F\left(d_{j}(n-1, n)\right) \leq F\left(d_{j}(n, n+1)\right) \\
& +L \theta\left(d_{j}(n+1, n-1)\right) \\
\Longrightarrow & \tau \leq F(1)-F(1)+L \theta(2) \Longrightarrow \tau \leq L \theta(2) .
\end{aligned}
$$

Hence, for any $0<\tau<2 / 3$ and $L=1$, (13) is satisfied and thus $(T, g)$ is a $\theta$-extended $\mathscr{W} \mathscr{J}$-edge contraction and $\theta$-extended $\mathscr{W} \mathscr{C}$-edge contraction. However, the pair $(T, g)$ is neither an $\xi$-extended $\mathscr{J} \mathscr{W}$-edge contraction pair nor an $\xi$-extended $\mathscr{C} \mathscr{W}$-contraction pair. If we take $g$ to be the identity mapping, then $T$ is a $\theta$-extended $\mathscr{J} \mathscr{W}$ -edge contraction mapping and $\theta$-extended $\mathscr{C} \mathscr{W}$-edge contraction mapping. However, again $T$ is none of Wardowski's F-contraction, Wardowski and Van Dung's F- weak contraction, and Ciric's quasicontraction.

\section{Main Results}

We start by proving the following main theorems:

Theorem 18. Suppose $\left(X^{j}, d_{j}\right)$ be endowed with a graph $G$ satisfying transitivity property, and the following conditions hold for $T, g: X^{j} \longrightarrow X^{j}$.

(a) $\left(g x_{0}^{j}, T x_{0}^{j}\right) \in E(G)$ for some $x_{0}^{j} \in X^{j}$

(b) $T$ is g-edge preserving

(c) $(T, g)$ is an $\theta$-extended $\mathscr{C} \mathscr{W}$-edge contraction pair of mappings

$(d)\left(d_{1}\right)$ There exists an edge-complete subset $M^{j}$ of $X^{j}$ for which $T\left(X^{j}\right) \subseteq M^{j} \subseteq g\left(X^{j}\right)$

$\left(d_{2}\right)$ One of the following conditions holds:

(i) $T$ is g-edge continuous

(ii) $T$ and $g$ are continuous

(iii) $\left.E(G)\right|_{X^{j}}$ satisfies property $(*)$

Then, the pair $(T, g)$ has a coincidence point.

Proof. In view of the assumption (a), we have $\left(g x_{0}^{j}, T x_{0}^{j}\right) \epsilon$ $E(G)$. If $T x_{0}^{j}=g x_{0}^{j}$, then $x_{0}$ is a coincidence point of $(T, g)$, i.e., $\operatorname{Coin}(T, g) \neq \phi$, and there is nothing to prove. Assume 
that $T x_{0}^{j} \neq g x_{0}^{j}$; then, since $T\left(X^{j}\right) \subseteq g\left(X^{j}\right)$, there exists $x^{j}{ }_{1} \in$ $X^{j}$ such that $g x_{1}^{j}=T x_{0}^{j}$.

Similarly, there is $x_{2}^{j} \in X^{j}$ such that $g x_{2}^{j}=T x_{1}^{j}$ with $(g$ $\left.x_{1}^{j}, g x_{2}^{j}\right) \in E(G)$ and consequently $\left(T x_{0}^{j}, T x_{1}^{j}\right) \in E(G)$. Inductively, one can construct a sequence $\left\{x_{n}^{j}\right\} \subseteq X^{j}$ such that

$$
g x_{n+1}^{j}=T x_{n}^{j}, \text { for all } n \in \mathbb{N}_{0},
$$

with

$$
\left(g x^{j}{ }_{n}, g x_{n+1}^{j}\right) \in E(G) \text { for all } n \in \mathbb{N}_{0}
$$

and consequently, as $T$ is $g$-edge preserving,

$$
\left(T x_{n}^{j}, T x_{n+1}^{j}\right) \in E(G)
$$

Now, if $T x_{n_{0}}^{j}=T x_{n_{0}}^{j}$ for some $n_{0} \in \mathbb{N}_{0}$, then $x_{n_{0}}$ is a coincidence point $(T, g)$ and we are done. Assume that $T x_{n}{ }_{n} \neq$ $T x_{n+1}^{j}$, for all $n \in \mathbb{N}_{0}$. On using (21), (22), (23), and condition $(c)$, we have

$$
\begin{aligned}
\tau+F\left(d\left(g x_{n}^{j}, g x_{n+1}^{j}\right)\right) & =\tau+F\left(\left(d\left(T x_{n-1}^{j}, T x_{n}^{j}\right)\right)\right) \\
& \leq F\left(M\left(x_{n-1}^{j}, x_{n}^{j}\right)\right)+L \theta\left(d\left(g x_{n}^{j}, T x_{n-1}^{j}\right)\right) .
\end{aligned}
$$

Now,

$$
\begin{aligned}
M\left(x_{n-1}^{j}, x_{n}^{j}\right) & =\max \left\{d_{j}\left(g x_{n-1}^{j}, g x_{n}^{j}\right), d_{j}\left(g x_{n-1}^{j}, T x_{n-1}^{j}\right), d_{j}\left(g x_{n}^{j}, T x_{n}^{j}\right), \frac{d_{j}\left(g x_{n-1}^{j}, T x_{n}^{j}\right)+d_{j}\left(g x_{n}^{j}, T x_{n-1}^{j}\right)}{2}\right\} \\
& =\max \left\{d_{j}\left(g x_{n-1}^{j}, g x_{n}^{j}\right), d_{j}\left(g x_{n}^{j}, g x_{n+1}^{j}\right)\right\}, \\
\theta\left(d\left(g x_{n}^{j}, T x_{n-1}^{j}\right)\right) & =\theta\left(d\left(g x_{n}^{j}, g x_{n}^{j}\right)\right)=0 .
\end{aligned}
$$

Thus, we get

$\tau+F\left(d\left(g x_{n}^{j}, g x_{n+1}^{j}\right)\right) \leq F\left(\max \left\{d_{j}\left(g x_{n-1}^{j}, g x_{n}^{j}\right), d_{j}\left(g x_{n}^{j}, g x_{n+1}^{j}\right)\right\}\right)$,

i.e.,

$\left.\left.F\left(d\left(g x_{n}^{j}, g x_{n+1}^{j}\right)\right)<\tau+F\left(d\left(g x_{n}^{j}, g x_{n+1}^{j}\right)\right) \leq F\left(d\left(g x_{n-1}^{j}, g x_{n}^{j}\right)\right)\right\}\right)$.

Since $F$ is nondecreasing, we get $d\left(g x_{n}^{j}, g x_{n+1}^{j}\right)<d(g$ $\left.\left.x_{n-1}^{j}, g x_{n}^{j}\right)\right)$. This further means that $d_{j}\left(x_{n}^{j}, x_{n+1}^{j}\right) \longrightarrow \delta \geq 0$ as $n \longrightarrow+\infty$. If $\delta>0$, we obtain from (27) that

$$
F(\delta+) \leq \tau+F(\delta+) \leq F(\delta+),
$$

which is a contradiction. Hence, $\lim _{n \longrightarrow+\infty} d_{j}\left(x_{n}^{j}, x_{n+1}^{j}\right)=0$. Suppose the sequence $\left\{g x_{n}^{j}\right\}$ is not a Cauchy sequence. By Lemma 11 , there exist $\xi>0$ and sequences $\left\{n_{k}\right\}$ and $\left\{p_{k}\right\}$ in $\mathbb{N}$ such that $n_{k}>p_{k}>k$, such that the sequences $d_{j}\left(x_{n_{k}}^{j}, x_{p_{k}}^{j}\right)$ and $d_{j}\left(x_{n_{k}+1}^{j}\right.$, $\left.x_{p_{k}+1}^{j}\right)$ tend to be $\xi^{+}$, as $k \longrightarrow+\infty$. By (27) we get

$$
\tau+F\left(\xi^{+}+\right) \leq F\left(\xi^{+}+\right)
$$

which is a contradiction. So sequence $\left\{g x_{n}^{j}\right\}$ is a Cauchy sequence.

By (21) and (22), $\left\{g x_{n}^{j}\right\}$ is an edge-preserving Cauchy sequence in $T\left(X^{j}\right) \subset M^{j}$, and since $M^{j}$ is edge-complete, there exists $y^{j} \in M^{j}$ such that $\left\{g x^{j}{ }_{n}\right\} \longrightarrow y^{j}$. As $M^{j} \subseteq g\left(X^{j}\right)$, there exists $u^{j} \in X^{j}$ such that $y^{j}=g u^{j}$. Hence, on using (21), we obtain

$$
\lim _{n \longrightarrow \infty} g x_{n}^{j}=\lim _{n \longrightarrow \infty} T x_{n}^{j}=g u^{j} .
$$

Now, suppose condition $\left(d_{2}(\mathrm{i})\right)$ is true. Using (22) and (30), we obtain

$$
\lim _{n \longrightarrow \infty} T x_{n}^{j}=T u^{j} .
$$

By (30) and (31), we have

$$
T u^{j}=g u^{j} .
$$

Suppose condition $\left(d_{2}(\mathrm{ii})\right)$ is true. By Lemma 10 , there is $S \subseteq X^{j}$ for which $g(S)=g\left(X^{j}\right)$ and $g: S \longrightarrow S$ is one-one. Consider the function $f: g(S) \longrightarrow g\left(X^{j}\right)$ given by

$$
f(g s)=T s(g s \in g(S), s \in S) .
$$

As $g: S \longrightarrow X^{j}$ is one-one and $T\left(X^{j}\right) \subseteq g\left(X^{j}\right), f$ is well- 
defined. Since $T$ and $g$ are continuous, $f$ is also continuous by condition $\left(d_{1}\right)$ of the hypothesis $T\left(X^{j}\right) \subseteq M^{j} \subseteq g(S)$. Thus, we have $\left\{x_{n}^{j}\right\} \subseteq S$ and $u^{j} \in S$. Therefore,

$$
T u^{j}=f\left(g u^{j}\right)=f\left(\lim _{n \longrightarrow \infty} g x_{n}^{j}\right)=\lim _{n \longrightarrow \infty} f\left(g x_{n}^{j}\right)=\lim _{n \longrightarrow \infty} T x_{n}^{j}=g u^{j} .
$$

Suppose condition $\left(d_{2}(\mathrm{iii})\right)$ is true; that is, $\left.E(G)\right|_{X^{j}}$ satisfied Property $(*)$. Since $\left\{g x_{n}^{j}\right\} \subseteq X$, it follows that $\{g$ $\left.x_{n}^{j}\right\}$ is $\left.E(G)\right|_{X^{j}}$-preserving (due to (22)) and $\left\{g x_{n}^{j}\right\} \longrightarrow$ $g u^{j}$ (by (30)) and so we have a subsequence $\left\{g x_{n_{k}}^{j}\right\} \subseteq\{g$ $x_{n}^{j}$ \} such that

$$
\left.\left(g x_{n_{k}}^{j}, g \mathcal{u}^{j}\right) \in E(G)\right|_{X}, \quad \text { for all } k \in \mathbb{N}_{0}
$$
have

Using (35) and condition (b) of the hypothesis, we

$$
\left.\left(T x_{n_{k}}^{j}, T u^{j}\right) \in E(G)\right|_{X^{j}} \subseteq S, \quad \text { for all } k \in \mathbb{N}_{0} .
$$

Now, let $P^{j}=\left\{k \in \mathbb{N}: T x_{n_{k}}^{j}=T u\right\}$.

If $P^{j}$ is finite, then $\left\{T x_{n_{k}}^{j}\right\}$ has a subsequence $\left\{T x_{n_{k_{i}}}^{j}\right\}$ such that $T x_{n_{k_{i}}}^{j} \neq T u$ for all $i \in \mathbb{N}$. Also, $\left.\left(g x_{n_{k_{i}}}^{j}, g u^{j}\right) \in E(G)\right|_{X} \subset E(G)$. Thus, we have

$$
\begin{gathered}
\tau+F\left(d\left(T x_{n_{k_{i}}}^{j}, T u^{j}\right)\right) \leq F\left(M\left(x_{n_{k_{i}}}^{j}, u^{j}\right)\right)+L \theta\left(d\left(g u^{j}, T x_{n_{k_{i}}}^{j}\right)\right), \\
M\left(x_{n_{k_{i}}}^{j}, u^{j}\right)=\max \left\{d_{j}\left(g x_{n_{k_{i}}}^{j}, g u^{j}\right), d_{j}\left(g x_{n_{k_{i}}}^{j}, T x_{n_{k_{i}}}^{j}\right), d_{j}\left(g u^{j}, T u^{j}\right), \frac{d_{j}\left(g x_{n_{k_{i}}}^{j}, T u^{j}\right)+d_{j}\left(g u^{j}, T g x_{n_{k_{i}}}^{j}\right)}{2}\right\} .
\end{gathered}
$$

Letting $i \longrightarrow \infty$, we obtain $M\left(x_{n_{k_{i}}}^{j}, u^{j}\right)=d_{j}\left(g u^{j}, T u^{j}\right)$ and $\theta\left(d\left(g u^{j}, T x_{n_{k_{i}}}^{j}\right)\right)=0$. Thus, we get

$$
\tau+F\left(d_{j}\left(g u^{j}, T u^{j}\right)\right) \leq F\left(d\left(g u^{j}, T u^{j}\right)\right),
$$

which is a contradiction. Hence, $P^{j}$ is not finite. Thus, $P^{j}$ is infinite and so $\left\{T x_{n_{k}}^{j}\right\}$ has a subsequence $\left\{T x_{n_{k_{i}}}^{j}\right\}$ such that $T x_{n_{k_{i}}}^{j}=T u^{j}$ for all $i \in \mathbb{N}$. Thus, $\lim _{i \longrightarrow \infty} T x_{n_{k_{i}}}^{j}=T u^{j}$. As $\lim _{n \longrightarrow \infty} T x_{n}^{j}=g u^{j}$ (by (30)), we get $T u^{j}=g u^{j}$.

Theorem 19. If, in addition to hypothesis $(a)-(d)$ of Theorem 18, we assume the following:

(i) For all $u^{j}, v^{j} \in \operatorname{Coin}(T, g)$,

$$
\begin{aligned}
d_{j}\left(T u^{j}, T v^{j}\right) & >0 \Longrightarrow \tau+F\left(d_{j}\left(T u^{j}, T v^{j}\right)\right) \\
& \leq \mathscr{F}\left(M^{j}\left(u^{j}, v^{j}\right)\right)+L \theta\left(d_{j}\left(g u^{i}, T u^{i}\right)\right),
\end{aligned}
$$

(ii) One of $T$ or $g$ is one-one

(iii) $T$ and $g$ are weakly compatible

then $(T, g)$ has a unique common fixed point.

Proof. In view of Theorem 18, the set $\operatorname{Coin}(T, g)$ is nonempty. Let $u^{j}, v^{j} \in \operatorname{Coin}(T, g)$. If $d_{j}\left(T u^{j}, v^{j}\right)=0$, then we have $T u^{j}=g u^{j}=g v^{j}=T v^{j}$, and hence, $u^{j}=v^{j}$ as one of $T$ and $g$ is one-one. Otherwise, using condition (39), we obtain

$$
\begin{aligned}
\tau+F\left(d\left(T u^{j}, T v^{j}\right)\right) & \leq F\left(d\left(g u^{j}, g v^{j}\right)\right)+L \theta\left(d\left(g u^{j}, T u^{j}\right)\right) \\
& =F\left(d\left(T u^{j}, T v^{j}\right)\right),
\end{aligned}
$$

which is a contradiction. So the coincidence point of $T$ and $g$ is unique.

Let $u^{j}$ be the unique coincidence point of $T$ and $g$, and let $z^{j} \in X$ such that $z^{j}=T u^{j}=g u^{j}$. As $T$ and $g$ are weakly compatible, we have $T z^{j}=T g u^{j}=g T u^{j}=g z^{j}$. Thus, $z^{j}$ is a coincidence point of $T$ and $g$. By the uniqueness of the coincidence point, we conclude $u^{j}=z^{j}$; that is, $u$ is a common fixed point of the pair $(T, g)$ which is indeed unique. as the coincidence point of $T$ and $g$ is unique.

Remark 20. If we replace condition $(d)$ of Theorem 18 with the following alternate condition:

$\left(d^{*}\right)\left(d_{1}^{*}\right)$ There exists a subset $Y^{j}$ of $X^{j}$ such that $T\left(X^{j}\right)$ $\subseteq g\left(X^{j}\right) \subseteq Y^{j}$ and $Y^{j}$ is edge-complete

$\left(d_{2}^{*}\right)(T, g)$ is an edge-compatible pair

$\left(d_{3}^{*}\right) T$ and $g$ are edge-continuous

the conclusions of Theorems 18 and 19 still hold.

Proof. Clearly, $\left\{g x_{n}^{j}\right\}$ is an edge-preserving Cauchy sequence in $Y^{j}$, and by edge-completeness of $Y$, we get $v^{j}$ $\in Y^{j}$ such that 


$$
\lim _{n \longrightarrow \infty} g x_{n}^{j}=v^{j}
$$

and then, by (21), we have

$$
\lim _{n \longrightarrow \infty} T x_{n}^{j}=v^{j} .
$$

Using the edge continuity of $g$ and $T$, we also have

$$
\begin{aligned}
& \lim _{n \longrightarrow \infty} T\left(g x_{n}^{j}\right)=T\left(\lim _{n \longrightarrow \infty} g x^{j}{ }_{n}\right)=T v^{j}, \\
& \lim _{n \longrightarrow \infty} g\left(T x_{n}^{j}\right)=g\left(\lim _{n \longrightarrow \infty} T x_{n}^{j}\right)=g v^{j} .
\end{aligned}
$$

Then, by edge-compatibility of $g$ and $T$, we get

$$
\lim _{n \rightarrow \infty} d\left(g T x_{n}^{j}, T g x_{n}^{j}\right)=0 .
$$

Finally from (44), (45), and (43), we get

$d\left(g v^{j}, T v^{j}\right)=d\left(\lim _{n \longrightarrow \infty} g T x^{j}{ }_{n}, \lim _{n \longrightarrow \infty} T g x^{j}{ }_{n}\right)=\lim _{n \longrightarrow \infty}\left(g T x_{n}^{j}, T g x_{n}^{j}\right)=0$.

Hence, $v^{j}$ is a coincidence point of the pair $(T, g)$.

Remark 21. Since every $\xi$-extended contraction mapping is a $\theta$-extended contraction, the conclusions of Theorems 18 and 19 remain true for an edge theoretic $\xi$-extended $\mathscr{C} \mathscr{W}$-contraction pair of mappings also.

On setting $g=I$ in Theorem 18, we deduce the following corresponding fixed-point result.

Theorem 22. Let $(M, d)$ be a metric space endowed with a directed graph $G$ and $T: M \longrightarrow M$. Assume that the following conditions are fulfilled:

(a) There exists $x_{0} \in M$ such that $\left(x_{0}, T x_{0}^{j}\right) \in E(G)$

(b) $T$ is edge-preserving

(c) $T$ is a $\theta$-extended $\mathscr{C} \mathscr{W}$-edge contraction mapping

(d) $\left(d_{1}\right)$ There exists a subset $X$ of $M$ such that $T(M) \subseteq X$ and $X$ is edge-complete

$\left(d_{2}\right)$ One of the following conditions is satisfied:

(i) $T$ is edge-continuous

(ii) $\left.E(G)\right|_{X}$ satisfies Property $(*)$

Then, Thas a fixed point.

Example 6. Let $\left\{X^{j}, d_{j}\right\}, E(G), T$, and $g$ be as in Example 5. Then, we have the following:

(1) $(g 0, T 0) \in E(G)$

(2) $T$ is $g$-edge-preserving. In fact, we see that $\left(g x^{j}, g y^{j}\right)$ $\in E(G)$ implies either $x^{j}=n, y^{j}=n$ or $x^{j}=n, y^{j}=n$
+1 . If $n=0$, then $(T 0, T 0) \in E(G)$ and $(T 0, T 1) \epsilon$ $E(G)$. If $n=1$, then $(T 1, T 1) \in E(G)$ and $(T 1, T 2)$ $\in E(G)$. If $n=k>1$, then $(T k, T k) \in E(G)$ and $(T$ $k, T(k+1))=(k-1, k) \in E(G)$

(3) $(T, g)$ is a $\theta$-extended $\mathscr{C} \mathscr{W}$-edge contraction mapping

(4) $T\left(X^{j}\right) \subset g\left(X^{j}\right)$

(5) $T$ is $g$-edge-continuous

Thus, all conditions of Theorem 18 are satisfied and 0 is a coincidence point of $T$ and $g$. Moreover, we see that $T$ and $g$ satisfy conditions (i), (ii) ( $g$ is one-one), and (iii) of Theorem 19 , and 0 is the unique common fixed point of $T$ and $g$.

Remark 23 (an open problem). Prove Theorems 18, 19, and 22 for $\xi$-extended $\mathscr{C} \mathbb{Q} \mathscr{W}$-contraction mappings.

\section{Application to Nonlinear Integral Equations}

Consider the Banach space $M=C([0,1], R)$ of all continuous functions $x:[0,1] \longrightarrow R$ equipped with norm

$$
\|x\|=\max _{s \in[0,1]}|x(s)|
$$

Define a metric $d_{j}$ on $M$ by $d_{j}\left(x^{j}, y^{j}\right)=\left\|x^{j}-y^{j}\right\|$ for all $x^{j}, y^{j} \in M$. Then, $\left(M, d_{j}\right)$ is a complete metric space.

In this section, we show the applicability of Theorem 19 by investigating the existence and uniqueness of a solution for the following nonlinear integral equation of Volterra type:

$$
\begin{aligned}
x^{j}(s)= & \int_{0}^{\mu(s)} K\left(s, v,\left(x^{j}\right)(\eta(v))\right) d v \\
& +\int_{0}^{\sigma(s)} J\left(s, v,\left(x^{j}\right)(\zeta(v))\right) d v+f(s), s \in[0,1],
\end{aligned}
$$

where $K, J:[0,1] \times[0,1] \times R \longrightarrow R, f:[0,1] \longrightarrow R$, and $\mu$, $\sigma, \eta, \zeta:[0,1] \longrightarrow[0,1]$

Definition 24. A lower solution for (48) is a function $x \in M$ such that

$$
\begin{aligned}
x^{j}(s) \leq & \int_{0}^{\mu(s)} K\left(s, v,\left(x^{j}\right)(\eta(v))\right) d v \\
& +\int_{0}^{\sigma(s)} J\left(s, v,\left(x^{j}\right)(\zeta(v))\right) d v+f(s), s \in[0,1] .
\end{aligned}
$$

Definition 25. An upper solution for (48) is a function $x \in M$ such that

$$
\begin{aligned}
x^{j}(s) \geq & \int_{0}^{\mu(s)} K\left(s, v,\left(x^{j}\right)(\eta(v))\right) d v \\
& +\int_{0}^{\sigma(s)} J\left(s, v,\left(x^{j}\right)(\zeta(v))\right) d v+f(s), s \in[0,1] .
\end{aligned}
$$


Consider the operator $T: M \longrightarrow M$ defined by

$$
\begin{aligned}
T\left(x^{j}(s)\right)= & \int_{0}^{\mu(s)} K\left(s, v,\left(x^{j}\right)(\eta(v))\right) d v \\
& +\int_{0}^{\sigma(s)} J\left(s, v,\left(x^{j}\right)(\zeta(v))\right) d v+f(s), \text { for all } x \in M .
\end{aligned}
$$

Then, $x^{j}$ is a fixed point of the operator $T$ if and only if it is a solution of the integral equation (48).

Let

$$
\begin{aligned}
M^{\diamond}\left(x^{j}, y^{j}\right) & =\max \left\{\left|x^{j}-y^{j}\right|,\left|x^{j}-T\left(x^{j}(s)\right)\right|,\left|y^{j}-T\left(y^{j}(s)\right)\right|, \frac{\left|x^{j}-T\left(y^{j}(s)\right)\right|+\left|y^{j}-T\left(x^{j}(s)\right)\right|}{2}\right\}, \\
\left\|M^{\diamond}\left(x^{j}, y^{j}\right)\right\| & =\max \left\{\left\|x^{j}-y^{j}\right\|,\left\|x^{j}-T\left(x^{j}(s)\right)\right\|,\left\|y^{j}-T\left(y^{j}(s)\right)\right\|, \frac{\left\|x^{j}-T\left(y^{j}(s)\right)\right\|+\left\|y^{j}-T\left(x^{j}(s)\right)\right\|}{2}\right\} .
\end{aligned}
$$

Theorem 26. Assume that $K$ and $J$ are nondecreasing in the third variable, $\mu(t)+\sigma(t) \leq 1$ for all $t \in[0,1]$, and the following conditions hold:
There exists $\tau>0$ such that

$$
\begin{aligned}
\left|K\left(s, v, g x^{j}\right)-K\left(s, v, g y^{j}\right)\right| & \leq \frac{M^{\diamond}\left(x^{j}, y^{j}\right)}{\left\|M^{\diamond}\left(x^{j}, y^{j}\right)\right\|\left\{\tau-\left(\left(L\left\|y^{j}-T\left(x^{j}(s)\right)\right\|\right) /\left(1+\left\|y^{j}-T\left(x^{j}(s)\right)\right\|\right)\right)\right\}+1}, \\
\left|J\left(s, v, g x^{j}\right)-J\left(s, v, g y^{j}\right)\right| & \leq \frac{M^{\diamond}\left(x^{j}, y^{j}\right)}{\left\|M^{\diamond}\left(x^{j}, y^{j}\right)\right\|\left\{\tau-L\left\|y^{j}-T\left(x^{j}(s)\right)\right\| / 1+\left|y^{j}-T\left(x^{j}(s)\right)\right|\right\}+1},
\end{aligned}
$$

for all $s, v \in[0,1], x^{j}, y^{j} \in M$ with $x^{j}(s) \leq y^{j}(s)$ and $L \geq 0$. If (48) has a lower solution, e.g., $x_{0}^{j}(s)$, then a solution exists for the integral equation (48).

Proof. Consider the graph $G$ in $M$, with edges $E(G)$ given by

$$
E(G)=\left\{\left(x^{j}, y^{j}\right) \in M \times M: x^{j}(s) \leq y^{j}(s)\right\} .
$$

For any $\left(x^{j}, y^{j}\right) \in E(G)$, we have (for all $s \in[0,1]$ )

$$
\begin{aligned}
T\left(x^{j}(s)\right)= & \int_{0}^{\mu(s)} K\left(s, v,\left(x^{j}\right)(\eta(v))\right) d v \\
& +\int_{0}^{\sigma(s)} J\left(s, v,\left(x^{j}\right)(\zeta(v))\right) d v+f(s) \\
\leq & \int_{0}^{\mu(s)} K\left(s, v,\left(y^{j}\right)(\eta(v))\right) d v \\
& +\int_{0}^{\sigma(s)} J\left(s, v,\left(y^{j}\right)(\zeta(v))\right) d v+f(s) \\
= & T\left(y^{j}(s)\right),
\end{aligned}
$$

which shows that $\left(T x^{j}, T y^{j}\right) \in E(G)$. Thus, $T$ is edgepreserving. Now, for all $\left(x^{j}, y^{j}\right) \in E(G)$ and $s \in[0,1]$, we have

$$
\begin{aligned}
& \left|T\left(x^{j}(s)\right)-T\left(y^{j}(s)\right)\right| \leq \int_{0}^{s} \mid\left(K\left(s, v,\left(x^{j}\right)(\eta(v))\right)\right. \\
& \left.-K\left(s, v,\left(y^{j}\right)(\eta(v))\right)\right)\left|d v+\int_{0}^{s}\right|\left(J\left(s, v,\left(x^{j}\right)(\eta(v))\right)-J\left(s, v,\left(y^{j}\right)(\eta(v))\right)\right) \mid d v \\
& \leq \int_{0}^{\mu(s)} \frac{M^{\circ}\left(x^{j}, y^{j}\right)}{M^{\circ}\left(x^{j}, y^{j}\right)\left\{\tau-\left(L\left\|y^{j}-T\left(x^{j}(s)\right)\right\| /\left(1+\left|y^{j}-T\left(x^{j}(s)\right)\right|\right)\right)\right\}+1} d v \\
& +\int_{0}^{\sigma(s)} \frac{M^{o}\left(x^{j}, y^{j}\right)}{M^{\circ}\left(x^{j}, y^{j}\right)\left\{\tau-\left(L\left\|y^{j}-T\left(x^{j}(s)\right)\right\| /\left(1+\left|y^{j}-T\left(x^{j}(s)\right)\right|\right)\right)\right\}+1} d v \\
& \leq \int_{0}^{\mu(s)} \frac{\max _{s \in 0,1]} M^{o}\left(x^{j}, y^{j}\right)}{\left\|M^{\circ}\left(x^{j}, y^{j}\right)\right\|\left\{\tau-\left(L\left\|y^{j}-T\left(x^{j}(s)\right)\right\| \mid\left(1+\left|y^{j}-T\left(x^{j}(s)\right)\right|\right)\right)\right\}+1} d v \\
& +\int_{0}^{\sigma(s)} \frac{\max _{s \in[0,1]} M^{o}\left(x^{j}, y^{j}\right)}{M^{o}\left(x^{j}, y^{j}\right)\left\{\tau-\left(L \| y^{j}-T\left(x^{j}(s)\right)|| /\left(1+\left|y^{j}-T\left(x^{j}(s)\right)\right|\right)\right)\right\}+1} d v \\
& \leq \frac{\left\|M^{\circ}\left(x^{j}, y^{j}\right)\right\|}{\left\|M^{\circ}\left(x^{j}, y^{j}\right)\right\|\left\{\tau-\left(L\left\|y^{j}-T\left(x^{j}(s)\right)\right\| /\left(1+\left|y^{j}-T\left(x^{j}(s)\right)\right|\right)\right)\right\}+1} \int_{0}^{\mu(s)} d v \\
& +\frac{\left\|M^{\circ}\left(x^{j}, y^{j}\right)\right\|}{M^{o}\left(x^{j}, y^{j}\right)\left\{\tau-\left(L\left\|y^{j}-T\left(x^{j}(s)\right)\right\| /\left(1+\left|y^{j}-T\left(x^{j}(s)\right)\right|\right)\right)\right\}+1} \int_{0}^{\sigma(s)} d v \\
& =\frac{\left\|M^{\circ}\left(x^{j}, y^{j}\right)\right\|}{M^{\circ}\left(x^{j}, y^{j}\right)\left\{\tau-\left(L\left\|y^{j}-T\left(x^{j}(s)\right)\right\| /\left(1+\left|y^{j}-T\left(x^{j}(s)\right)\right|\right)\right)\right\}+1}(\mu(s)+\sigma(s)) \\
& \leq \frac{\left\|M^{o}\left(x^{j}, y^{j}\right)\right\|}{M^{o}\left(x^{j}, y^{j}\right)\left\{\tau-\left(L\left\|y^{j}-T\left(x^{j}(s)\right)\right\| /\left(1+\left|y^{j}-T\left(x^{j}(s)\right)\right|\right)\right)\right\}+1} .
\end{aligned}
$$


Taking the supremum, we get

$\|T(x)-T(y)\| \leq \frac{\left\|M^{\circ}\left(x^{j}, y^{j}\right)\right\|}{M^{\circ}\left(x^{j}, y^{j}\right)\left\{\tau-\left(L\left\|y^{j}-T\left(x^{j}(s)\right)\right\| /\left(1+\left|y^{j}-T\left(x^{j}(s)\right)\right|\right)\right)\right\}+1}$,

or

$$
\tau+\frac{1}{\left\|M^{\diamond}\left(x^{j}, y^{j}\right)\right\|} \leq \frac{1}{\|T(x)-T(y)\|}+\frac{L\left\|y^{j}-T\left(x^{j}(s)\right)\right\|}{1+\left|y^{j}-T\left(x^{j}(s)\right)\right|},
$$

or

$$
\tau-\frac{1}{\|T(x)-T(y)\|} \leq \frac{-1}{\left\|M^{\diamond}\left(x^{j}, y^{j}\right)\right\|}+\frac{L\left\|y^{j}-T\left(x^{j}(s)\right)\right\|}{1+\left|y^{j}-T\left(x^{j}(s)\right)\right|} .
$$

That is,

$$
\tau-\frac{1}{d_{j}\left(T x^{j}, T y^{j}\right)} \leq \frac{-1}{\left\|M^{j}\left(x^{j}, y^{j}\right)\right\|}+\frac{L d_{j}\left(y^{j}, T x^{j}(s)\right) \|}{1+d_{j}\left(y^{j}, T x^{j}(s)\right)} .
$$

Thus, inequality (13) is satisfied with $F(\alpha)=-1 / \alpha$ and $\theta(\beta)=\beta /(1+\beta)$, so that $\lambda=\sup _{t>0} \theta(t)=1$. Also, by Definition 24, we have $\left(x_{0}^{j}, T x_{0}^{j}\right) \in E(G)$. Therefore, all the assumptions of Theorem 22 are satisfied, and thus, problem (48) has a solution.

Theorem 27. Assume that $K$ is nonincreasing in the third variable and there exists $\tau>0$ such that

$$
\left|K\left(s, v, g x^{j}\right)-K\left(s, v, g y^{j}\right)\right| \leq \frac{\left|g x^{j}-g y^{j}\right|}{\tau\left\|g x^{j}-g y^{j}\right\|+1},
$$

for all $s, v \in[0,1]$ and $x, y \in M$. Then, the existence of an upper solution of the integral equation (48) ensures the existence of a solution of (48).

Proof. Define set $E(G)$ of edges on $M$ by

$$
E(G)=\{(x, y) \in M \times M: x(s) \geq y(s)\} .
$$

Now, following the steps of the proof of Theorem 26 with an analogous procedure, one can check that all the hypotheses of Theorem 22 are validated, and thus, Theorem 22 ensures the existence of a unique solution of the integral equation (48).

We now furnish a numerical example to validate the hypothesis of Theorem 27.

Example 7. Consider the function $x \in M$ defined by $x(s)=$ $s^{2}, s \in[0,1]$. We show that this function is an upper solution in $M$ for the following integral equation:

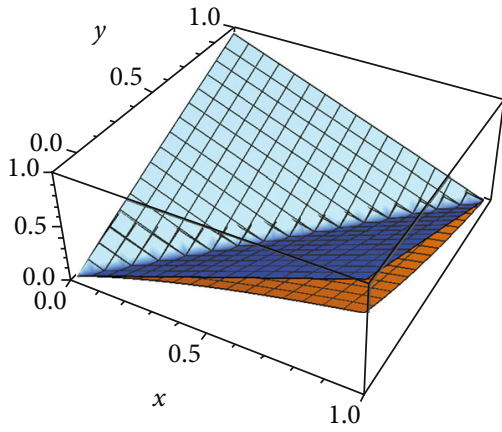

$$
\frac{|\log (1+x)-\log (1+y)|}{1+0.01|x-y|}
$$

Figure 1: Inequality in (66).

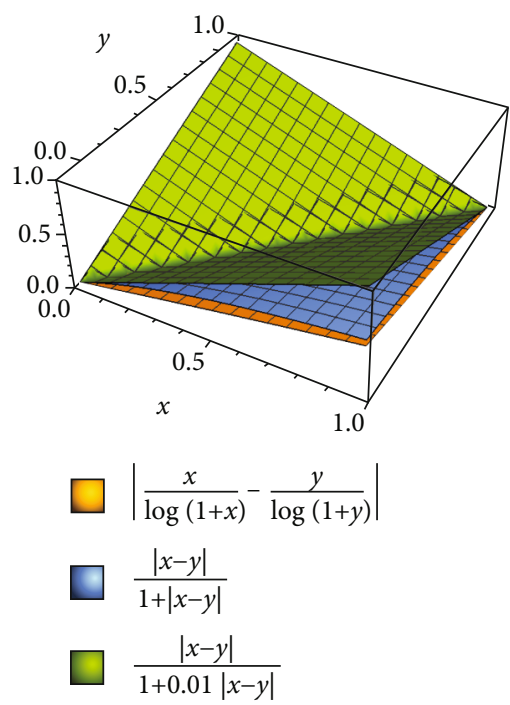

FIGURE 2: Inequality in (67).

$$
\begin{aligned}
x(s)= & -\frac{1}{2} s+2 s^{2}+\arctan \left(\frac{1}{2} s\right)-3 \arctan \left(\frac{1}{2} s^{2}\right)-\frac{1}{2} s^{2} \ln \left(1+\frac{1}{4} s^{4}\right) \\
& +\int_{0}^{s^{2} / 2} \ln (1+x(v)) d v+\int_{0}^{s / 2} \frac{x(v)}{1+x(v)} d v, \quad s \in[0,1] .
\end{aligned}
$$

Finally, we see that $x_{u}(s)=s^{2}-\arctan \left(s^{2} / 2\right)$ is the unique solution of (63).

Proof. Define the operator $T: M \longrightarrow M$ as

$$
\begin{aligned}
T x(s)= & -\frac{1}{2} s+2 s^{2}+\arctan \left(\frac{1}{2} s\right)-3 \arctan \left(\frac{1}{2} s^{2}\right) \\
& -\frac{1}{2} s^{2} \ln \left(1+\frac{1}{4} s^{4}\right)+\int_{0}^{s^{2} / 2} \ln (1+x(v)) d v \\
& +\int_{0}^{s / 2} \frac{x(v)}{1+x(v)} d v, \quad s \in[0,1] .
\end{aligned}
$$


Now, set $K(s, v, x(v))=\ln (1+x(v)), J(s, v, x(v))=x(v)$ $/(1+x(v)), \mu(s)=(1 / 2) s^{2}, \sigma(s)=(1 / 2) s, f(s)=-(1 / 2) s+2 s^{2}$ $+\arctan ((1 / 2) s)-3 \arctan \left((1 / 2) s^{2}\right)-(1 / 2) s^{2} \ln (1+(1 / 4)$ $\left.s^{4}\right)$, and $\tau \leq 0.01$. We observe the following:

(i) Both the functions $K(s, v, x(v))=\ln (1+x(v))$ and $J(s, v, x(v))=x(v) /(1+x(v))$ are nondecreasing in the third variable

(ii) By actual computation, we have

$$
\begin{gathered}
\int_{0}^{s^{2} / 2} \ln (1+x(v)) d v=-s^{2}+2 \arctan \left(\frac{1}{2} s^{2}\right)+\frac{1}{2} s^{2} \ln \left(1+\frac{1}{4} s^{4}\right), \quad s \in[0,1], \\
\int_{0}^{s / 2} \frac{x(v)}{1+x(v)} d v=\frac{1}{2} s-\arctan \left(\frac{1}{2} s\right), \quad s \in[0,1] .
\end{gathered}
$$

(iii) $s^{2} \geq-(1 / 2) s+2 s^{2}+\arctan ((1 / 2) s)-3 \arctan ((1 / 2)$ $\left.s^{2}\right)-(1 / 2) s^{2} \ln \left(1+(1 / 4) s^{4}\right)+\int_{0}^{s^{2} / 2} \ln (1+x(v)) d v$ $+\int_{0}^{s / 2} x(v) /(1+x(v)) d v, s \in[0,1]$ so that $x(s)=s^{2}$ is an upper solution for (63)

(iv) The following inequalities hold true for all $x, y$ $\in[0,1]$ (see Figures 1 and 2):

$$
\begin{aligned}
|\ln (1+x)-\ln (1+y)| & \leq \frac{|x-y|}{1+0.01|x-y|}, \\
\left|\frac{x}{1+x}-\frac{y}{1+y}\right| & \leq \frac{|x-y|}{1+|x-y|} \leq \frac{|x-y|}{1+0.01|x-y|} .
\end{aligned}
$$

Furthermore, using the nondecreasing function $s \mapsto s$ / $(1+0.01 s)$, we have

$$
\begin{aligned}
|\ln (1+x)-\ln (1+y)| & \leq \frac{|x-y|}{1+0.01|x-y|} \\
& \leq \frac{\max _{s \in[0,1]}|x-y|}{1+0.01 \max _{s \in[0,1]}|x-y|} \\
& =\frac{\|x-y\|}{1+0.01\|x-y\|} .
\end{aligned}
$$

Similarly, for all $x, y \in[0,1]$, we have

$$
\left|\frac{x}{1+x}-\frac{y}{1+y}\right| \leq \frac{\|x-y\|}{1+0.01\|x-y\|}
$$

Hence, all the conditions of Theorem 27 are satisfied. It is evident that the integral equation (63) has a unique solution $x_{u} \in M$ defined by $x_{u}(s)=s^{2}-\arctan \left(s^{2} / 2\right)$.

\section{Data Availability}

No data were used to support this study.

\section{Conflicts of Interest}

The authors declare that they have no conflicts of interest.

\section{References}

[1] D. Boyd and J. S. W. Wong, "On nonlinear contractions," Proceedings of American Mathematical Society, vol. 20, no. 2, pp. 458-464, 1969.

[2] L. B. Ciric, "A generalization of Banach's contraction principle," Proceedings of American Mathematical Society, vol. 45, no. 2, pp. 267-273, 1974.

[3] R. Gubran, M. Imdad, I. A. Khan, and W. M. Alfaqih, "Ordertheoretic common fixed point results for F-contractions," Bulletin of Mathematical Analysis and Applications, vol. 10, no. 1, pp. 80-88, 2018.

[4] J. J. Nieto and R. Lòpez, "Contractive mapping theorems in partially ordered sets and applications to ordinary differential equations," Order, vol. 22, no. 3, pp. 223-239, 2005.

[5] H. Piri and P. Kumam, "Some fixed point theorems concerning F-contraction in complete metric spaces," Fixed Point Theory and Applications, vol. 2014, no. 1, 2014.

[6] A. C. M. Ran and M. C. B. Reurings, "A fixed point theorem in partially ordered sets and some applications to matrix equations," Proceedings of the American Mathematical Society, vol. 132, pp. 1435-1443, 2004.

[7] S. B. Nadler, "Multi-valued contraction mappings," Pacific Journal of Mathematics, vol. 30, no. 2, pp. 475-488, 1969.

[8] D. Wardowski, "Fixed points of a new type of contractive mappings in complete metric spaces," Fixed Point Theory and Applications, vol. 94, 6 pages, 2012.

[9] D. Wardowski and N. Van Dung, "Fixed points of F-weak contractions on complete metric spaces," Demonstratio Mathematica, vol. 47, no. 1, pp. 146-155, 2014.

[10] G. Durmaz, G. Minak, and I. Altun, "Fixed points of ordered F-contractions," Hacettepe Journal of Mathematics and Statistics, vol. 1, no. 45, pp. 15-21, 2016.

[11] S. Sawangsup, W. Sawangsup, A. Francisco, and R. L. De Heirro, "Fixed point theorems for F_\mathfrak $\{\mathrm{R}\}$-contractions with applications to solution of nonlinear matrix equations," Journal of Fixed Point Theory and Applications, vol. 19, no. 3, pp. 1711-1725, 2017.

[12] M. Imdad, Q. Khan, W. M. Alfaqih, and R. Gubran, "A relation theoretic $(\mathrm{F}, \mathrm{R})$-contraction principle with applications to matrix equations," Bulletin of Mathematical Analysis and Applications, vol. 10, no. 1, pp. 1-12, 2018.

[13] W. M. Alfaqih, M. Imdad, R. Gubran, and I. A. Khan, "Relation-theoretic coincidence and common fixed point results under $(\mathrm{F}, \mathrm{R})_{\mathrm{g}}$-contractions with an application," Fixed Point Theory and Applications, vol. 12, 18 pages, 2019.

[14] R. Espinola and W. A. Kirk, "Fixed point theorems in R-trees with applications to graph theory," Topology and its Applications, vol. 153, no. 7, pp. 1046-1055, 2006.

[15] J. Jachymski, "The contraction principle for mappings on a metric space with a graph," Proceedings of the American Mathematical Society, vol. 136, no. 4, pp. 1359-1373, 2008. 
[16] W. M. Alfaqih, R. Gubran, and M. Imdad, "Coincidence and common fixed point results under generalized $(\mathrm{A}, \mathrm{S})_{\mathrm{f}}$-contractions," Filomat, vol. 32, no. 7, pp. 2651-2666, 2018.

[17] R. Batra and S. Vashistha, "Fixed points of an F-contraction on metric spaces with a graph," International Journal of Computer Mathematics, vol. 91, no. 12, pp. 2483-2490, 2014.

[18] I. Beg, A. R. Butt, and S. Radojevic, "The contraction principle for set valued mappings on a metric space with a graph," Computers \& Mathematcs with Applications, vol. 60, no. 5, pp. 1214-1219, 2010.

[19] F. Bojor, "Fixed point of $\varphi$-contraction in metric spaces endowed with a graph," Annals of the University of CraiovaMathematics and Computer Science Series, vol. 37, no. 4, pp. 85-92, 2010.

[20] F. Bojor, "Fixed point theorems for Reich type contractions on metric spaces with a graph," Nonlinear Analysis, vol. 75, no. 9, pp. 3895-3901, 2012.

[21] F. Bojor, "Fixed points of Kannan mappings in metric spaces endowed with a graph," Versita, vol. 20, no. 1, pp. 31-40, 2012.

[22] C. Chifu and G. Petrusel, "New results on coupled fixed point theory in metric spaces endowed with a directed graph," Fixed Point Theory and Applications, vol. 151, 11 pages, 2014.

[23] G. Prasad, R. C. Dimri, and A. Bartwal, "Coincidence theorems in new generalized metric spaces under locally g-transitive binary relation," Journal of the Indian Mathematical Society, vol. 85, no. 3-4, pp. 396-410, 2018.

[24] G. Prasad, "Fixed points of Kannan contractive mappings in relational metric spaces," The Journal of Analysis, vol. 29, no. 3, pp. 669-684, 2021.

[25] R. H. Haghi, S. Rezapour, and N. Shahzad, "Fixed points of Gtype quasi-contractions on graphs," Abstract and Applied Analysis, vol. 2013, Article ID 167530, 5 pages, 2013.

[26] S. Aleksić, Z. Mitrovic, and S. T. Radenovíc, "Picard sequences in b-metric spaces," Fixed Point Theory, vol. 21, no. 1, pp. 35$46,2020$. 